\title{
Manual Therapy as a Management of Cervical Radiculopathy: A Systematic Review
}

\author{
Sergio Borrella-Andrés $\left(\mathbb{D},{ }^{1,2}\right.$ Isabel Marqués-García, ${ }^{1,2}$ María Orosia Lucha-López, \\ Pablo Fanlo-Mazas ${ }^{(D)}$, ${ }^{1,2}$ Mar Hernández-Secorún ${ }^{D},{ }^{1,2}$ Albert Pérez-Bellmunt $(\mathbb{D}, 3,4$ \\ José Miguel Tricás-Moreno, ${ }^{1,2}$ and César Hidalgo-García ${ }^{1,2}$
}

\author{
${ }^{1}$ Health Sciences Faculty, University of Zaragoza, Zaragoza 50009, Spain \\ ${ }^{2}$ Physiotherapy Research Unit, University of Zaragoza, Zaragoza 50009, Spain \\ ${ }^{3}$ Department of Basic Sciences, Faculty of Medicine and Health Sciences, Universitat Internacional de Catalunya, \\ Sant Cugat del Vallés 08195, Spain \\ ${ }^{4}$ ACTIUM Functional Anatomy Group, Universitat Internacional de Catalunya, Sant Cugat del Vallés 08195, Spain
}

Correspondence should be addressed to Mar Hernández-Secorún; marhsecorun@unizar.es

Received 3 March 2021; Revised 3 May 2021; Accepted 27 May 2021; Published 4 June 2021

Academic Editor: Tsung-Hsun Hsieh

Copyright ( $\odot 2021$ Sergio Borrella-Andrés et al. This is an open access article distributed under the Creative Commons Attribution License, which permits unrestricted use, distribution, and reproduction in any medium, provided the original work is properly cited.

\begin{abstract}
Background. Cervical radiculopathy is defined as a disorder involving dysfunction of the cervical nerve roots characterised by pain radiating and/or loss of motor and sensory function towards the root affected. There is no consensus on a good definition of the term. In addition, the evidence regarding the effectiveness of manual therapy in radiculopathy is contradictory. Objective. To assess the effectiveness of manual therapy in improving pain, functional capacity, and range of motion in treating cervical radiculopathy with and without confirmation of altered nerve conduction. Methods. Systematic review of randomised clinical trials on cervical radiculopathy and manual therapy, in PubMed, Web of Science, Scopus, PEDro, and Cochrane Library Plus databases. The PRISMA checklist was followed. Methodological quality was evaluated using the PEDro scale and RoB 2.0. tool. Results. 17 clinical trials published in the past 10 years were selected. Manual therapy was effective in the treatment of symptoms related to cervical radiculopathy in all studies, regardless of the type of technique and dose applied. Conclusions. This systematic review did not establish which manual therapy techniques are the most effective for cervical radiculopathy with electrophysiological confirmation of altered nerve conduction. Without this confirmation, the application of manual therapy, regardless of the protocol applied and the manual therapy technique selected, appears to be effective in reducing chronic cervical pain and decreasing the index of cervical disability in cervical radiculopathy in the short term. However, it would be necessary to agree on a definition and diagnostic criteria of radiculopathy, as well as the definition and standardisation of manual techniques, to analyse the effectiveness of manual therapy in cervical radiculopathy in depth.
\end{abstract}

\section{Introduction}

Cervical radiculopathy $(\mathrm{CR})$ can be defined as a disorder involving dysfunction of the cervical nerve roots, commonly presenting with pain radiating from the neck towards the root affected [1]. Commonly, there is no agreement on the definition, given that it has also been defined as neck and shoulder pain combined with loss of sensory and motor function [2,3]. Nevertheless, Thoomes et al. [4] proposed a new definition of $\mathrm{CR}$ as a radiating pain in the arm with motor, reflex, and/or sensory changes (such as paraesthesia or numbness), provoked by neck posture(s) and/or movement(s).

The incidence of CR has been established to be between 63.5 and 107.3 per 100.000 people per year [5], with the C6 and $\mathrm{C} 7$ segments being the most affected [6]. As for the treatment given, there are 2 main approaches: the conservative option and the surgery. Clinical guidelines from 2011 and 2018 recommend exercise, manual therapy, and nonsteroid anti-inflammatory drugs (NSAIDs) as the first line of treatment $[7,8]$. If this first treatment option gives no relief within 
4 to 8 weeks [9], analgesic/anti-inflammatory drugs would be injected, recurring to surgery if necessary. Taking this route normally depends on how severe the patient's symptoms are. Evidence has also been found that both the surgical and the conservative approaches produce improvements by 2 years, with no statistically significant differences between them [10]. Other authors concluded that evidence on manual therapy was inconclusive or low levelled, and one single intervention cannot be recommended [11-13].

Previous systematic reviews have provided indications that exercise is effective in treating CR, whether associated or not to manual therapy $[14,15]$, manual or mechanical traction [16], and high-velocity/low-amplitude manipulations [17]. All the reviews coincide in the need to establish a treatment protocol and carry out studies having larger samples. However, the Task Force on Neck Pain and Its Associated Disorders (TFNPAD) concluded that there was insufficient evidence for recommending appropriate treatment for CR [18]. Consequently, the evidence as to the effectiveness of manual therapy in radiculopathy, regardless of the region, is contradictory.

Besides, without uniform CR diagnosis criteria, one might compare treatments with samples possibly having motor and/or sensory disorders to others in which the individuals have pain without altered nerve conduction velocity, with prognoses that are probably different. Despite conflicting evidence at the use of nerve conduction as a diagnostic criterion $[7,19]$, it has shown moderate diagnostic accuracy [20] and moderate-excellent specificity for radiculopathy $[20,21]$. Guidelines from the American Association of Neuromuscular and Electrodiagnostic Medicine (AANEM, previously American Association of Electrodiagnostic Medicine when published) recommend that for an optimal evaluation of a patient with suspected radiculopathy, a needle EMG screen of a sufficient number of muscles and at least one motor and one sensory nerve conduction study (NCS) should be performed in the involved limb [22]. NCSs are necessary to exclude polyneuropathy [22]. To date, no systematic review has been found that includes the idea of manual therapy effectiveness in $\mathrm{CR}$, in relation to altered nerve conduction measured with nerve conduction tests.

The objective of our systematic review is thus to verify the effectiveness of manual therapy in CR cases with and without confirmation of altered nerve conduction-using electromyography or electroneurography_in pain, disability, function, and range of movement, in comparison to other physiotherapy techniques used in the approach to CR.

\section{Materials and Methods}

2.1. Protocol and Recording. Our review was carried out in agreement with the PRISMA statement checklist [23] and the criteria of the Cochrane Handbook for Systematic Reviews of Interventions [24]. This systematic review was registered on the Open Science Framework digital platform: https://osf.io/zgdym/.

2.2. Information and Search Sources. Search strategy was developed following advice of an information specialist
(MHS) from the research group, with two-year experience in conducting systematic reviews. The following reference sources were searched: PubMed, Web of Science, Scopus, PEDro, and Cochrane Library Plus. The search terms were divided into 4 categories, as recommended by the Cochrane Back and Neck Group [25]. The first category was established to search for the types of studies to include: randomised controlled trial or controlled clinical trial. The second and third categories searched specifically for the condition (radiculopathy) and for manual therapy actions (musculoskeletal manipulations), while the fourth category served to limit the search to the cervical area (neck). These 4 searches were combined to obtain the results. Figure 1 presents a detailed search strategy, featuring the database search in PubMed, from which the rest of the databases were searched, adapting the searches to the requirements of each database.

2.3. Eligibility Criteria. The inclusion criteria were as follows: (1) randomised controlled clinical trials on humans; (2) patients with CR, identified and diagnosed by clinical criteria (arm, neck, scapular or periscapular pain and paresthesias, numbness and sensory changes, weakness, or abnormal deep tendon reflexes in the arm) [7] or by using references tests (shoulder abduction, Spurling's test) [7], electromyography or electroneurography, and the clinical criteria for radiculopathy diagnosis which had to be explained completely; (3) assessment of the effectiveness of manual therapy in CR, with no distinction between manipulations, mobilisations, and soft tissue treatment or combined with other techniques; (4) pain, disability, or Short Form-36 Health Survey (recommended by the North American Spinal Association in their CR guidelines [7]; (5) studies published in English, French, and Spanish; and (6) studies published in the past 10 years.

We excluded articles that (1) used another approach, except if they used a comparison to the manual therapy technique or were accompanied by it, and (2) with patients with neck and arm symptoms without a CR diagnosis.

2.4. Study Selection. Two researchers (IMG and SBA) selected the studies independently. If they disagreed on the study selection, the final decision was made by a third researcher ( $\mathrm{CHG}$ ).

2.5. Data Mining Process. Data on sample size, criteria for radiculopathy diagnosis, type of intervention, treatment protocol, follow-up, variables studied, and main results were included. With regard to the variables, the primary results, secondary results, and adverse effects were recorded.

2.6. Risk of Bias in the Individual Studies. Two independent researchers assessed methodological quality of the studies using the PEDro scale; there was a third researcher to settle cases of doubt or disagreement. The PEDro scale [26] evaluated 11 items, scoring each item as 1 or 0 , depending on whether the item fulfilled study criteria or not, respectively. External validity was assessed using Item 1, internal validity using Items 2 through 9, and interpretability of the results using Items 10 and 11 . The first item was not taken into account for the final score, which could be a maximum of 10 points. Each article was classified according to the score 


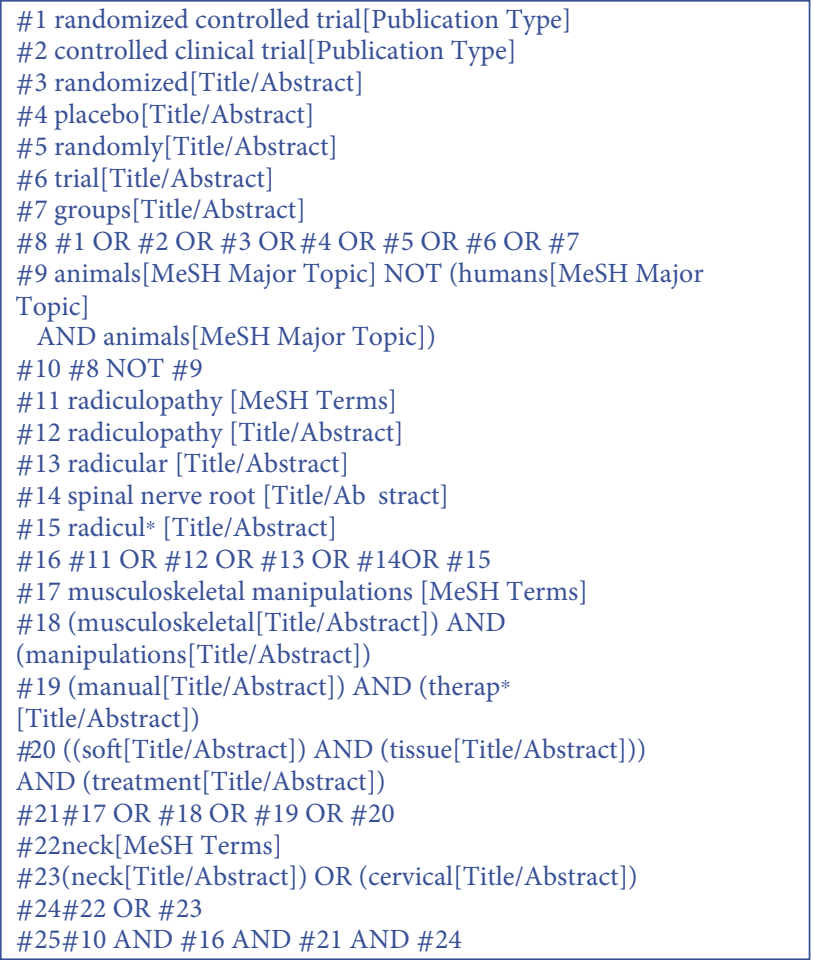

Figure 1: Search strategy.

obtained: "high quality" if its score was $\geq 6$, "moderate quality" with a score of 4-5, and "low quality" if the score was $<4$.

Also, the RoB2 tool was performed. It is the second version of the Cochrane tool to assess the risk of bias in clinical trials. The biases are evaluated in 5 domains: (1) randomization process, (2) effect of being assigned to intervention, (3) missing outcome data, (4) measurement of the outcome, and (5) reported results. Within each domain, 1 or more questions must be answered. These answers lead to the judgements of "low risk of bias," "some concerns," or "high risk of bias" [27].

\section{Results and Discussion}

3.1. Study Selection. The initial literature search yielded a total of 365 studies. After eliminating the duplicates, there were 211 articles left. Filtering on title and abstract yielded 22 articles for complete text reading, of which 5 were eliminated, because they did not meet any of the inclusion criteria. Seventeen studies were selected for final inclusion. The PRISMA flow diagram (Figure 2) illustrates the process.

3.2. Methodological Quality Assessment. The methodological quality of the trials included in the revision is summarised in Table 1, indicating if each of the PEDro Scale items is fulfilled or not. Of the 17 studies selected, 9 had an overall score of high quality [28-36]. Three studies had a moderate overall score [37-39] and 5 studies a low score [40-44].

Three studies [38, 40,41] did not assign patients randomly, and in the majority of the studies, patients were assigned with an inadequate concealment method. In four $[40,42-44]$ of the 15 studies, the intervention groups were not compared at the beginning of the study. All studies reported the lack of therapist blinding and the lack of patient blinding. Only 3 studies $[33,35,36]$ reported blinding of the assessors to the group assignment of the patients.

The RoB2 tools showed that the aspects with the worst methodological quality in all the studies are related to deviations from the intended intervention. Reported data and variable measurements seem to have the best methodological quality in all the studies, with $75 \%$ of the studies meeting these criteria (Figures 3(a) and 3(b)), even though the majority of studies had good/moderate methodological quality.

3.3. Clinical Trial Characteristics. Characteristics of the studies are presented in Table 2. There are a total of 1.183 patients. In all the studies, patients having symptoms compatible with a CR diagnosis who reported radiation of symptoms to an upper limb were discussed. CR diagnosis was established in all studies using the clinical findings, mentioning pain radiation or neural symptoms in the upper limb. None of the studies evaluated diagnosed radiculopathy using electromyography or electroneurography, and magnetic resonance was only used in 2 of the studies $[30,31]$ to reveal the possible cause of neural compression.

Nerve conduction velocity was analysed in just one study, using evoked potentials [31]. This test was performed as a measurement variable, not as a diagnostic criterion. In 8 of the studies assessed, the prediction rule formulated by Wainner et al. [45] was applied; this rule refers to a specificity of $94 \%-100 \%$ in diagnosing CR [30, 33, 35, 36, 38, 39, 43, 44].

The tools used to measure the study outcomes were similar in all the studies. The intensity of perceived pain was measured using a Visual Analogue Scale (VAS) in 8 studies $[30,33,35,36,38,39,43,44]$, while the Numeric Pain Rating Scale (NPRS) was used in the rest [30, 32, 34-36, 38, 39, 42, 43]. In 11 of the studies [28-32, 34-39, 42, 43], cervical disability was measured with the Neck Disability Index (NDI). As a secondary indicator of technique effectiveness, cervical mobility was measured in 6 of the studies [34, 35, 38-40, 43]. Likewise, quality of life was measured in just 1 of the studies [37], using the SF-36 Health Survey.

All the studies analysed reported a statistically significant improvement in the variables of pain and cervical disability index in the manual therapy intervention group, regardless of the protocol and the manual therapy technique applied. The manual therapy techniques of choice were divided among cervical manipulations, thoracic manipulations, cervical mobilisation towards the intervertebral foramen opening, cervical traction, and neural mobilisations. The techniques with the best results were those whose objective was to increase the intervertebral foramen area by transverse mobilisations in the indicated segments. These techniques had been applied with positive results in 4 of the studies $[28,29,41,43]$. However, one of them did not find differences with the control group [36]. Both the cervical and thoracic manipulation techniques yielded satisfactory results. Specifically, two studies on thoracic manipulation $[32,34]$ 


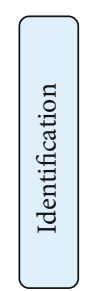

Records identified through database
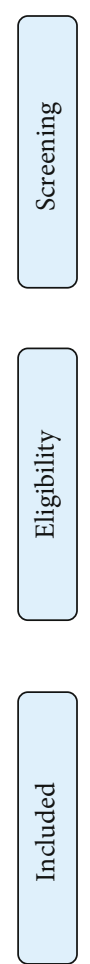

$$
\begin{aligned}
& \text { searching } \\
& (n=364)
\end{aligned}
$$

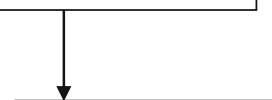

Records after duplicates removed

$$
(n=211)
$$

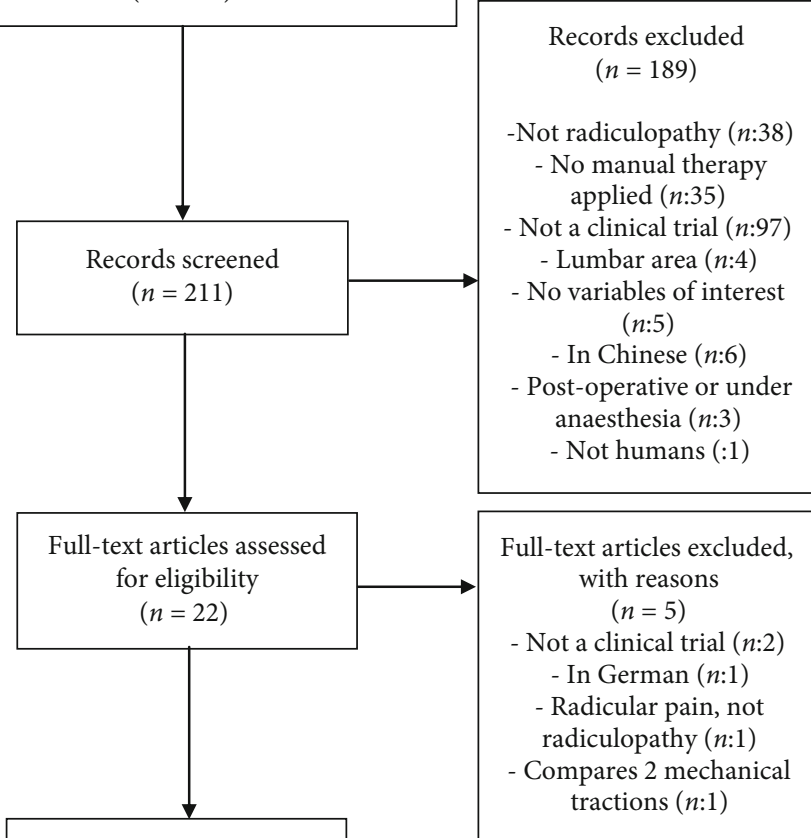

Studies included in qualitative synthesis $(n=17)$

Figure 2: PRISMA flow diagram.
Additional records identified through other sources $(n=1)$ 
TAble 1: Methodological quality according to the PEDro scale.

\begin{tabular}{llllllllllllcc}
\hline Study & 1 & 2 & 3 & 4 & 5 & 6 & 7 & 8 & 9 & 10 & 11 & Total & Quality \\
\hline Afzal (2019) & $\mathrm{X}$ & $\mathrm{X}$ & $\mathrm{X}$ & $\mathrm{X}$ & & & & $\mathrm{X}$ & & $\mathrm{X}$ & $\mathrm{X}$ & 6 & High \\
Bukhari (2016) & $\mathrm{X}$ & $\mathrm{X}$ & & & & & & $\mathrm{X}$ & & & $\mathrm{X}$ & 3 & Low \\
Cui (2017) & $\mathrm{X}$ & $\mathrm{X}$ & & $\mathrm{X}$ & & & & $\mathrm{X}$ & & $\mathrm{X}$ & $\mathrm{X}$ & 5 & Moderate \\
Eldesoky (2019) & $\mathrm{X}$ & $\mathrm{X}$ & $\mathrm{X}$ & $\mathrm{X}$ & & & & $\mathrm{X}$ & $\mathrm{X}$ & $\mathrm{X}$ & $\mathrm{X}$ & 7 & High \\
Hassan (2020) & $\mathrm{X}$ & $\mathrm{X}$ & & $\mathrm{X}$ & & & $\mathrm{X}$ & $\mathrm{X}$ & & $\mathrm{X}$ & & 5 & Moderate \\
Ibrahim (2019) & $\mathrm{X}$ & $\mathrm{X}$ & $\mathrm{X}$ & $\mathrm{X}$ & & & $\mathrm{X}$ & $\mathrm{X}$ & $\mathrm{X}$ & $\mathrm{X}$ & $\mathrm{X}$ & 8 & High \\
Khan K (2016) & $\mathrm{X}$ & $\mathrm{X}$ & & & & & & & & & $\mathrm{X}$ & 2 & Low \\
Kim D-G (2017) & $\mathrm{X}$ & & & $\mathrm{X}$ & & & $\mathrm{X}$ & $\mathrm{X}$ & $\mathrm{X}$ & $\mathrm{X}$ & 5 & Moderate \\
Kumar (2010) & $\mathrm{X}$ & & & & & & & & & $\mathrm{X}$ & $\mathrm{X}$ & 2 & Low \\
Langevin (2014) & $\mathrm{X}$ & $\mathrm{X}$ & $\mathrm{X}$ & $\mathrm{X}$ & & & $\mathrm{X}$ & $\mathrm{X}$ & $\mathrm{X}$ & $\mathrm{X}$ & $\mathrm{X}$ & 8 & High \\
Ojo Ojoawo (2016) & $\mathrm{X}$ & $\mathrm{X}$ & $\mathrm{X}$ & $\mathrm{X}$ & & & & $\mathrm{X}$ & $\mathrm{X}$ & $\mathrm{X}$ & $\mathrm{X}$ & 7 & High \\
Ojo Ojoawo (2018) & $\mathrm{X}$ & $\mathrm{X}$ & $\mathrm{X}$ & $\mathrm{X}$ & & & & $\mathrm{X}$ & $\mathrm{X}$ & $\mathrm{X}$ & $\mathrm{X}$ & 7 & High \\
Prabhakar (2011) & $\mathrm{X}$ & & & $\mathrm{X}$ & & & & & & $\mathrm{X}$ & $\mathrm{X}$ & 3 & Low \\
Shafique (2019) & $\mathrm{X}$ & $\mathrm{X}$ & & & & & & & & $\mathrm{X}$ & $\mathrm{X}$ & 3 & Low \\
Savva (2020) & $\mathrm{X}$ & $\mathrm{X}$ & $\mathrm{X}$ & $\mathrm{X}$ & & & $\mathrm{X}$ & $\mathrm{X}$ & $\mathrm{X}$ & $\mathrm{X}$ & $\mathrm{X}$ & 8 & High \\
Waqas (2016) & $\mathrm{X}$ & $\mathrm{X}$ & & $\mathrm{X}$ & & & & $\mathrm{X}$ & $\mathrm{X}$ & $\mathrm{X}$ & $\mathrm{X}$ & 6 & High \\
Young (2019) & $\mathrm{X}$ & $\mathrm{X}$ & $\mathrm{X}$ & $\mathrm{X}$ & & & & $\mathrm{X}$ & $\mathrm{X}$ & $\mathrm{X}$ & $\mathrm{X}$ & 7 & High \\
Mean & & & & & & & & & & & 5.5 & \\
\hline
\end{tabular}

\section{Discussion}

The objective of this review has been to ascertain the effectiveness of manual therapy in handling CR cases with and without confirmation of altered nerve conduction. In previous studies, the effectiveness of exercise in CR [14] and the interest in combining manual therapy with exercise has been demonstrated. The combination improved function, range of movement, and pain. However, it has been impossible to establish which manual therapy techniques are the most effective [15].

There is a lack of scientific research on applying manual therapy techniques (such as manipulations and mobilisations) in this pathology [16], as well as a lack of rigour in describing the manual therapy techniques used. A previous review showed a lack of evidence for the effectiveness of manual therapy in patients with CR [13], due to low-quality evidence or interventions studied only once. A noninvasive therapy in cervicobrachial pain has been previously revised with inconclusive evidence for the effectiveness of noninvasive management [11]. Considering previous reviews, several new studies have appeared and been included in this review, contributing to the new evidence regarding $\mathrm{CR}$ and manual therapy.

Degenerative signs in imaging tests need to be correlated with the clinical findings of the pathology for accurate CR diagnosis [46]. Therefore, our study has considered the relationship between patients with symptomatology compatible with $\mathrm{CR}$ and a medical diagnosis with imaging tests or nerve conduction tests.

All the studies analysed reported a statistically significant improvement in the variables pain and cervical disability index. Such improvements did not depend on the manual therapy protocol and the technique applied.
This review reveals the need to establish reference criteria for diagnosing "CR" cases, attempting to avoid a catch-all approach. None of the studies included performed electromyography or electroneurography to determine nerve conduction alteration, and only 2 of the studies carried out magnetic resonance imaging to demonstrate possible structural nerve root compromise [30,31]. It could be explained because of the conflicting evidence of using a conduction test as a diagnostic criteria $[7,19]$.

The studies include patients with symptomology similar to radicular conditions but not objectively cases of radiculopathy, due to the used diagnostic criteria. Myofascial trigger points, faceted radiated pain, and distal neural compressions might simulate similar symptomology.

Wainner et al. [45] formulate a prediction rule for diagnosing CR cases, achieving specificity of $94 \%-100 \%$ depending on the positivity of four diagnostic tests. However, none of these four tests involves loss of neural conduction in the form of loss of sensitivity and/or motor function and this prediction rule was based on a limited sample of 16 subjects with mild and moderate C6 and C7 CR and 57 controls. Schmid et al. [47] recommended adding pain and thermal sensibility to tactile and motor testing to include $\mathrm{A} \delta$ and $\mathrm{C}$ nerve fibers within the evaluation. A new standard definition of radiculopathy is needed, along with relevant diagnostic criteria.

Differentiation should be established between radiculalgia and radicular pain without altered nerve conduction velocity and radiculopathy involving loss of nerve conduction velocity (symptoms and/or signs of neural-origin sensory or motor loss). Similarly, Shacklock [48] defined CR neural management based on the intensity of the symptoms and the presence of neurological symptoms. This classification also guides professionals in the tests that have to be performed to alleviate or provoke patient symptoms more or less 


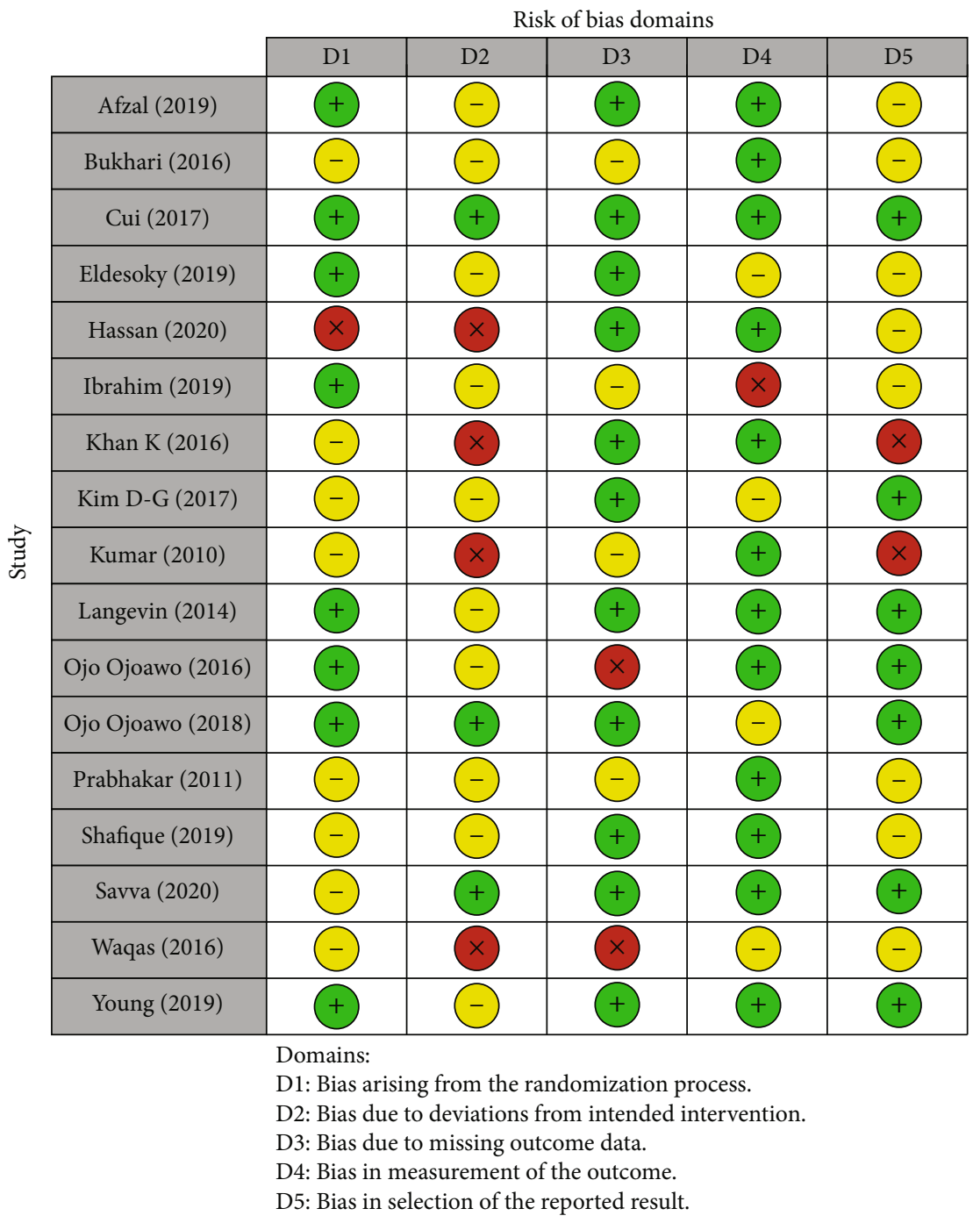

Judgement
(x) High
- Some concerns
(+) Low

(a)

Bias arising from the randomization process Bias due to deviations from intended intervention

Bias due to missing outcome data

Bias in measurement of the outcome Bias in selection of the reported result

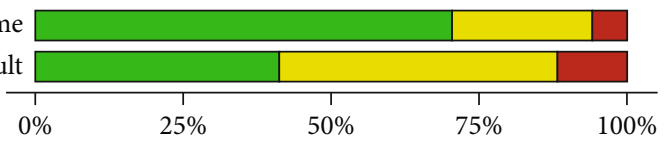

High

$\square$ Some concerns

$\square$ Low

(b)

Figure 3: (a) Summary of risk of bias 2.0. and (b) Risk of bias 2.0. graphs. 


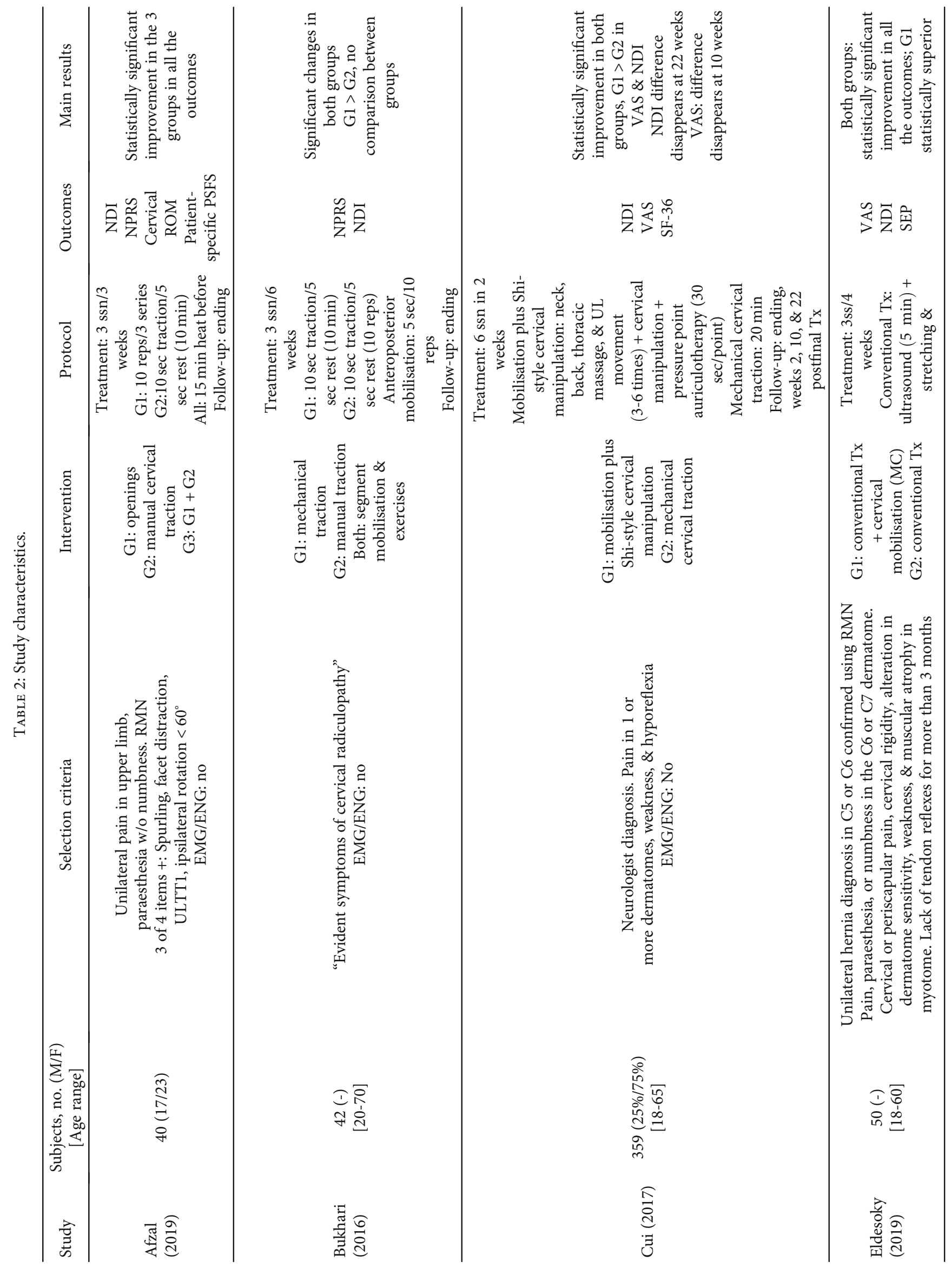




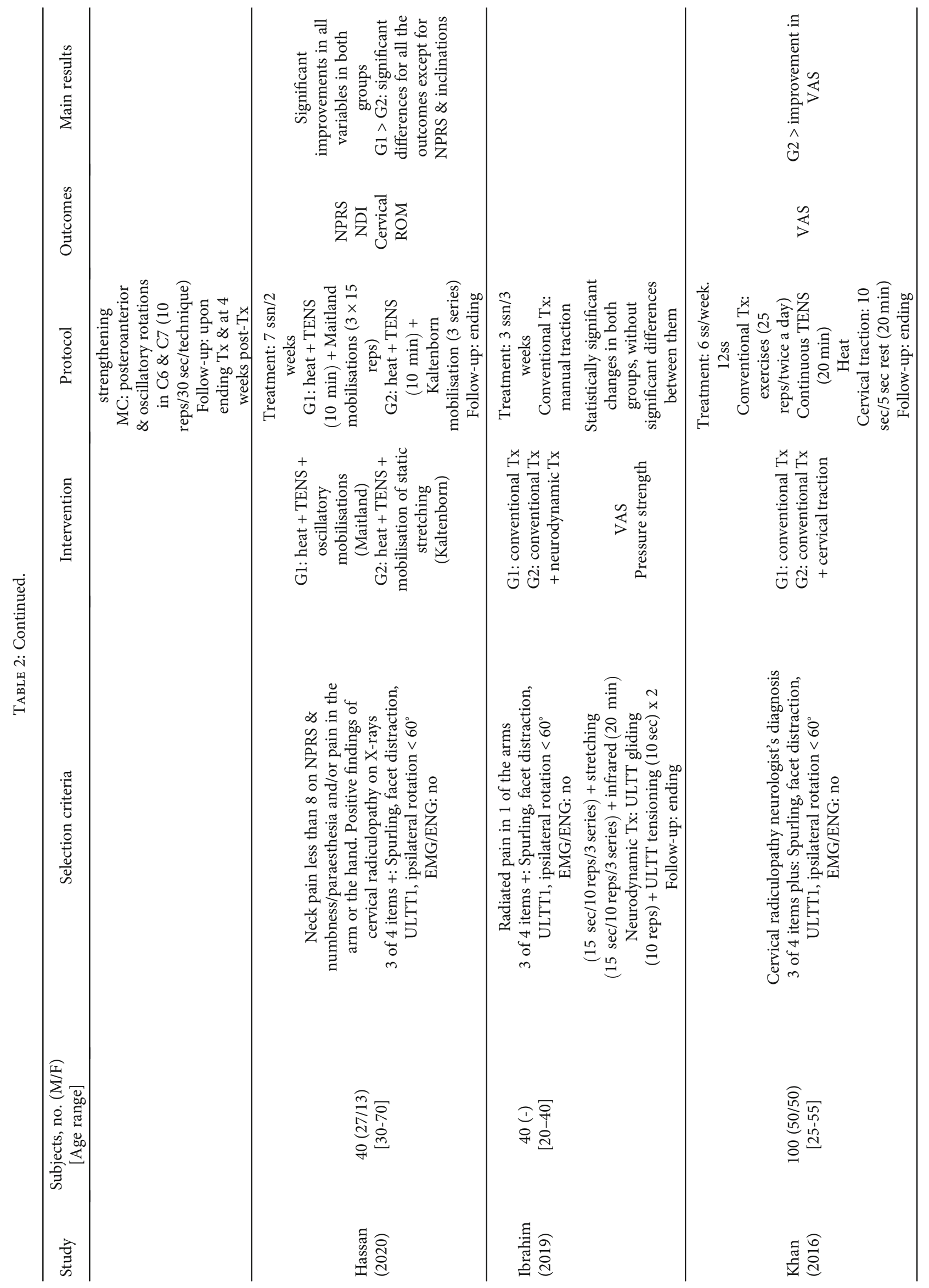




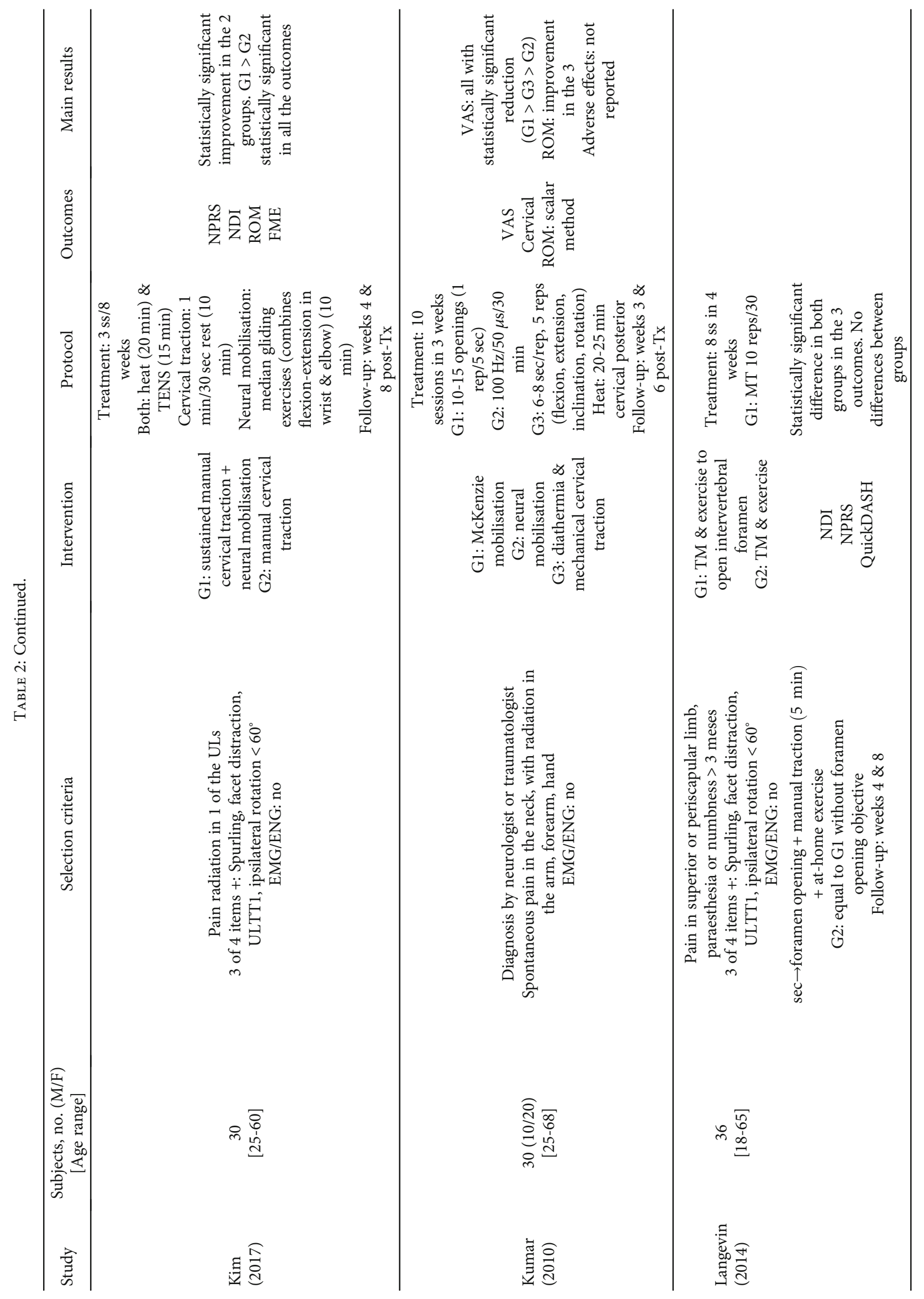




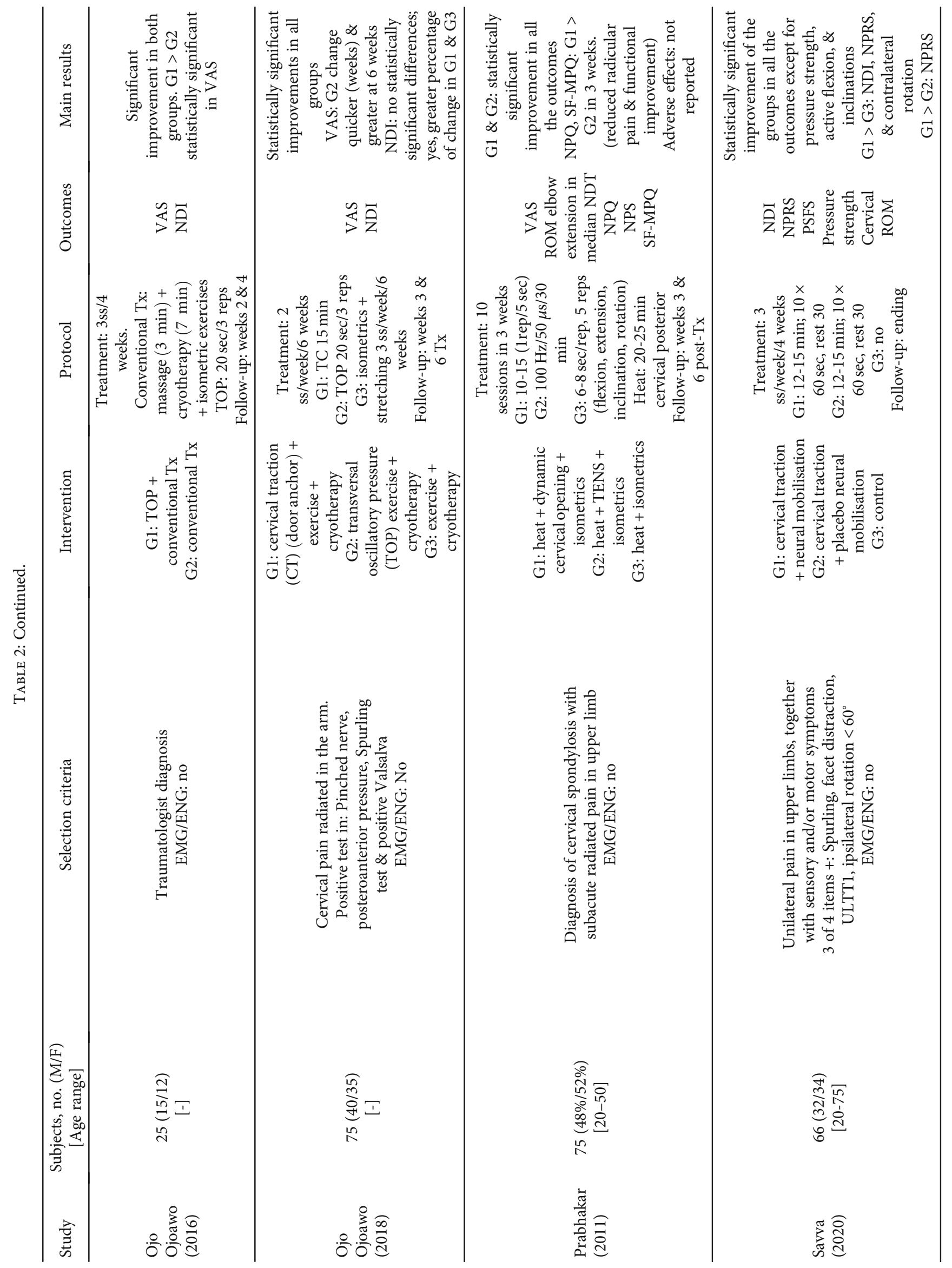




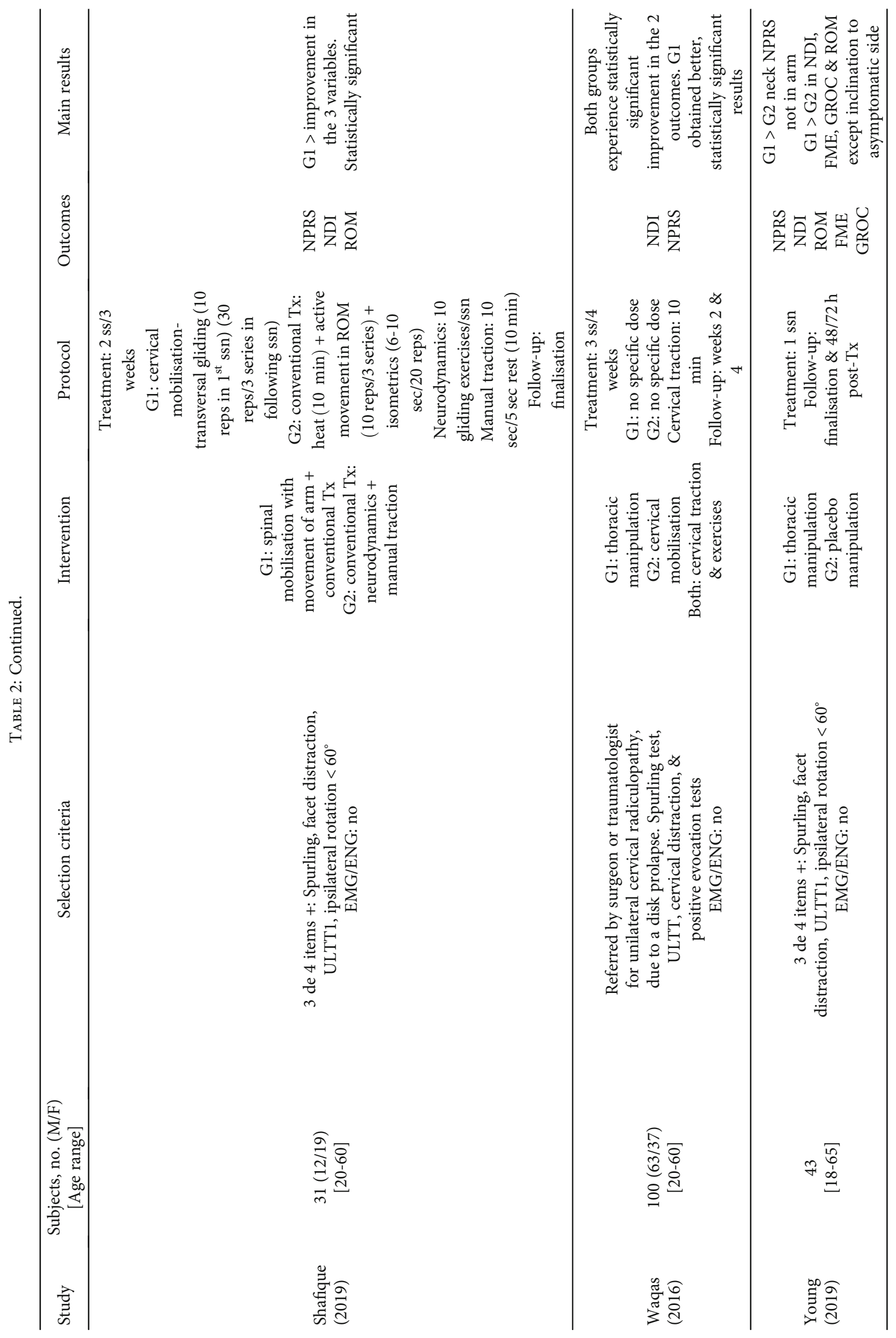


easily, as well as in the treatment techniques to use. For example, in patients classified as "Level 1," neural mobilisation techniques in tension might be contraindicated, because they may generate symptoms and could be counterproductive [48].

A combination of diagnostic criteria based on clinical findings compatible with radiculopathy, the prediction rule of Wainner et al. [45], clinical tests for various sensory and/or motor involvement, imaging tests such as magnetic resonance, and clinical tests for nerve conduction alteration should be performed. Thoomes et al. [19] propose a "combination of Spurling's, axial traction, and an Arm Squeeze test to increase the likelihood of a cervical radiculopathy, whereas a combined results of four negative neurodynamics tests and an Arm Squeeze test could be used to rule out the disorder." Nowadays, the usefulness of electrodiagnosis is still under debate; the NASS referred insufficient evidence to make a recommendation for or against EMG for patients with unclear diagnosis after clinical examination and MRI [7].

Given that there is symptomology presumably caused by radiculopathy, the techniques that offered the best results were those searching for the intervertebral foramen opening by using pressure or transverse oscillations. The neurodynamic techniques yielded the worst results; the parameters applied were a bit unclear, categorising the neurodynamic technique used as neural gliding exercises [38], mobilising only the elbow and wrist, and probably putting tension on the root and not performing a gliding exercise [48, 49]. Rade et al. [50] showed the conus medullaris displacement in all three planes when unilateral and bilateral SLR were applied. This represents a protective mechanism which preserves the spinal cord and lumbar neural roots from excessive strain. This could allow gliding treatment of the affected nerve root with the help of the mobilisation of the contralateral neurodynamic test. It would be interesting to observe the potential gliding effect of contralateral neurodynamic test on the nerve root on CR patients. A recent study has shown the effect of contralateral cervical lateral glide on CR patients [51], in which longitudinal median nerve excursion differed significantly between patients with CR and asymptomatic volunteers at baseline. This difference was no longer present after 3 months of conservative physiotherapy management.

This fact has led us to analyse the importance of establishing exact parameters for the technique applied and the technical development of its application. Because manual techniques are involved, the variables to control may depend on performance. Reflecting the performance parameters appropriately in the studies is recommended so that the results can be reproduced and extrapolated to the clinical setting. This is something at which the majority of studies has failed, naming the technique applied but not explaining it in detail. Our recommendation would be to avoid describing manual techniques in general, based only on the name of the used methodologies (Maitland, Kaltenborn) but without identifying precisely the technique. Our recommendation is to describe the procedures precisely using the TIDieR checklist to make sure they can be reproduced [52].
Treatment times and doses varied from some studies to others, within similar techniques. The mean was 2-3 sessions a week, applying approximately 8-12 sessions. There are no previous studies dealing with the number of sessions needed for conservative treatment of CR cases. These first guidelines in treatment times are considered interesting; in future studies, the mean treatment times with other approaches can be analysed to compare the time indication of each of the techniques.

As for the number of sessions proposed, thoracic manipulation might be one of the first techniques to apply because it has demonstrated beneficial results from a single session [34] and can be complemented with intervertebral foramen openings in following sessions. These 2 techniques showed the best results, obtained in 2 of the studies with a sufficient sample size [28, 41]. Cui et al. [37] showed high effectiveness in their study involving a multimodal approach to CR based on movement and acupuncture. These results can be considered for clinical application, seeking an approach to all the dysfunctional components found in the assessment by combining different manual therapy procedures.

The results presented in this review should be considered at short term, as the studies involve only short-term followup. Manual therapy can be proposed as a way to provide relief when the symptoms might be related to radiculopathy, but its long-term effectiveness has been impossible to establish.

The 3 indicators recommended by clinical guidelines for CR [7] have been analysed as primary variables. It would also be of interest for future studies to include the new functional impact scale, developed specifically for CR [53], as a measurement variable. After seeing the results of this systematic review, it is obvious that there is a need to make the definition of manual therapy techniques and the inclusion criteria more homogeneous and to provide greater detail in defining technique application parameters. This would make possible to advance in future meta-analyses and reviews, as it has been achieved in the approach to CR cases using exercise [14], by establishing more favourable parameters, techniques, and doses.

Our systematic review is strongly limited by the variability of the inclusion criteria of patients with CR and of the manual therapy techniques used, given that these have been very different or poorly explained in the majority of the studies. This means that it has been impossible to analyse the results specifically in the application of each of the techniques. Another limitation is that there may be references available besides the ones in Spanish, English, and French that have not been considered due to the language requirements established in our inclusion criteria. Finally, in the absence of a Cochrane Information Specialist, an information specialist of the group with a two-year experience was available that might not be able to fulfil all the functions.

\section{Conclusions}

We cannot state that manual therapy is effective in treating CR cases confirmed by EMG or ENG tests, due to a deficit 
in the diagnostic criteria used in the scientific literature. Without an EMG and ENG confirmation, a multimodal approach that includes manual therapy seems to be the most effective short-term approach. The methodological deficiencies and the lack of follow-ups in the studies have to be treated cautiously. It is therefore necessary to establish greater scientific evidence with high quality with larger sample sizes and longer follow-up times, in which initial treatment parameters are established for each of the manual therapy techniques. There is currently a lack of standardisation of diagnostic processes and of treatment in this pathology, even though manual therapy has been shown to be effective in dealing with CR cases having a diagnosis based on clinical criteria.

\section{Data Availability}

The data used to support the findings of this study are available from public databases, and more details also can be obtained from the corresponding author on request.

\section{Conflicts of Interest}

The authors declare that there is no conflict of interest regarding the publication of this paper.

\section{Authors' Contributions}

SB-A and IM-G performed strategy search. SB-A, CH-G, MOL-L, and MH-S wrote, translated, and adapted the article. The rest of the authors collaborated in the revision and correction of the review.

\section{References}

[1] J. M. Rhee, T. Yoon, and K. D. Riew, "Cervical radiculopathy," The Journal of the American Academy of Orthopaedic Surgeons, vol. 15, no. 8, pp. 486-494, 2007.

[2] S. Carette and M. G. Fehlings, "Cervical radiculopathy," New England Journal of Medicine, vol. 353, no. 4, pp. 392-399, 2005.

[3] D. L. Corey and D. Comeau, "Cervical radiculopathy," Medical Clinics of North America, vol. 98, no. 4, pp. 791-799, 2014.

[4] E. J. Thoomes, G. G. M. Scholten-Peeters, A. J. de Boer et al., "Lack of uniform diagnostic criteria for cervical radiculopathy in conservative intervention studies: A systematic review," European Spine Journal, vol. 21, no. 8, pp. 1459-1470, 2012.

[5] K. Radhakrishnan, W. J. Litchy, W. M. O'Fallon, and L. T. Kurland, "Epidemiology of cervical radiculopathy," Brain, vol. 117, no. 2, pp. 325-335, 1994.

[6] H. J. Kim, V. M. Nemani, C. Piyaskulkaew, S. R. Vargas, and K. D. Riew, "Cervical radiculopathy: incidence and treatment of 1,420 consecutive cases," Asian Spine Journal, vol. 10, no. 2, pp. 231-237, 2016.

[7] C. M. Bono, G. Ghiselli, T. J. Gilbert et al., "An evidence-based clinical guideline for the diagnosis and treatment of cervical radiculopathy from degenerative disorders," The Spine Journal, vol. 11, no. 1, pp. 64-72, 2011.

[8] M. J. Stochkendahl, P. Kjaer, J. Hartvigsen et al., "National clinical guidelines for non-surgical treatment of patients with recent onset low back pain or lumbar radiculopathy," European Spine Journal, vol. 27, no. 1, pp. 60-75, 2018.

[9] V. J. Alentado, D. Lubelski, M. P. Steinmetz, E. C. Benzel, and T. E. Mroz, "Optimal duration of conservative management prior to surgery for cervical and lumbar radiculopathy: a literature review," Global Spine Journal, vol. 4, no. 4, pp. 279-286, 2014.

[10] J. N. Weinstein, T. D. Tosteson, J. D. Lurie et al., "Surgical vs nonoperative treatment for lumbar disk herniation. The Spine Patient Outcomes Research Trial (SPORT): a randomized trial," Journal of the American Medical Association, vol. 296, no. 20, pp. 2441-2450, 2006.

[11] E. Salt, C. Wright, S. Kelly, and A. Dean, "A systematic literature review on the effectiveness of non-invasive therapy for cervicobrachial pain," Manual Therapy, vol. 16, no. 1, pp. 53-65, 2011.

[12] E. J. Thoomes, W. Scholten-Peeters, B. Koes, D. Falla, and A. P. Verhagen, "The effectiveness of conservative treatment for patients with cervical radiculopathy: a systematic review," The Clinical Journal of Pain, vol. 29, no. 12, pp. 1073-1086, 2013.

[13] E. J. Thoomes, "Effectiveness of manual therapy for cervical radiculopathy, a review," Chiropractic \& Manual Therapies, vol. 24, no. 1, pp. 1-11, 2016.

[14] L. Liang, M. Feng, X. Cui et al., "The effect of exercise on cervical radiculopathy: a systematic review and meta-analysis," Medicine (Baltimore), vol. 98, no. 45, article e17733, 2019.

[15] R. Boyles, P. Toy, J. Mellon, M. Hayes, and B. Hammer, "Effectiveness of manual physical therapy in the treatment of cervical radiculopathy: a systematic review," Journal of Manual \& Manipulative Therapy, vol. 19, no. 3, pp. 135142, 2011.

[16] A. Romeo, C. Vanti, V. Boldrini et al., "Cervical radiculopathy: effectiveness of adding traction to physical therapy-a systematic review and meta-analysis of randomized controlled trials," Physical Therapy, vol. 98, no. 4, pp. 231-242, 2018.

[17] R. J. Rodine and H. Vernon, "Cervical radiculopathy: a systematic review on treatment by spinal manipulation and measurement with the Neck Disability Index," Journal of the Canadian Chiropractic Association, vol. 56, no. 1, pp. 18-28, 2012.

[18] E. L. Hurwitz, E. J. Carragee, G. van der Velde et al., "Treatment of neck pain: noninvasive Interventions," European Spine Journal, vol. 17, no. S1, pp. 123-152, 2008.

[19] E. J. Thoomes, S. van Geest, D. A. van der Windt et al., "Value of physical tests in diagnosing cervical radiculopathy: a systematic review," The Spine Journal, vol. 18, no. 1, pp. 179189, 2018.

[20] P. Narayanaswami, T. Geisbush, L. Jones et al., "Critically reevaluating a common technique: accuracy, reliability, and confirmation bias of EMG," Neurology, vol. 86, no. 3, pp. 218-223, 2016.

[21] H. C. Tong, A. J. Haig, K. S. J. Yamakawa, and J. A. Miner, "Specificity of needle electromyography for lumbar radiculopathy and plexopathy in 55- to 79-year-old asymptomatic subjects," American Journal of Physical Medicine \& Rehabilitation, vol. 85, no. 11, pp. 908-912, 2006.

[22] American Association Of Electrodiagnostic Medicine, "Guidelines in electrodiagnostic medicine," Muscle Nerve, vol. 15, no. 2, pp. 229-253, 1992.

[23] D. Moher, A. Liberati, J. Tetzlaff, D. G. Altman, and and the PRISMA Group, "Reprint-preferred reporting items for 
systematic reviews and meta-analyses: the PRISMA statement," Physical Therapy, vol. 89, no. 9, pp. 873-880, 2009.

[24] J. P. Higgins and S. Green, "Cochrane Handbook for Systematic Reviews of Interventions," Cochrane Database of Systematic Reviews, pp. 1-639, 2011.

[25] A. D. Furlan, A. Malmivaara, R. Chou et al., "2015 updated method guideline for systematic reviews in the Cochrane Back and Neck Group," Spine (Phila Pa 1976), vol. 40, no. 21, pp. 1660-1673, 2015.

[26] R. Herbert, A. Moseley, C. Sherrington, and C. Maher, "Physiotherapy evidence database," Physiotherapy, vol. 86, no. 1, p. $55,2000$.

[27] J. A. C. Sterne, J. Savović, M. J. Page et al., "RoB 2: a revised tool for assessing risk of bias in randomised trials," BMJ, vol. 366, p. 14898, 2019.

[28] A. O. Ojoawo and A. D. Olabode, "Comparative effectiveness of transverse oscillatory pressure and cervical traction in the management of cervical radiculopathy: a randomized controlled study," Hong Kong Physiotherapy Journal, vol. 38, no. 2, pp. 149-160, 2018.

[29] A. O. Ojoawo, A. Olabode, O. Esan, A. Badru, S. Odejide, and B. Arilewola, "Transverse oscillatory pressure in management of cervical radiculopathy: a randomised controlled study," Hong Kong Physiotherapy Journal, vol. 34, pp. 1926, 2016.

[30] R. Afzal, M. Ghous, S. Shakil Ur Rehman, and T. Masood, "Comparison between manual traction, manual opening technique and combination in patients with cervical radiculopathy: randomized control trial," The Journal of the Pakistan Medical Association, vol. 69, no. 9, pp. 1237-1241, 2019.

[31] M. Eldesoky, H. Al Amer, E. Abutaleb, and A. Nassif, "Effect of cervical mobilization on nerve root function in cervical radiculopathy: a randomized trial," Bioscience Research, vol. 16, no. 4, pp. 3962-3972, 2019.

[32] S. Waqas, A. Ahmad, S. Ahmad, T. Shafi, and H. Shahid, "Comparison of Maitland thoracic spine manipulation versus Maitland cervical spine mobilization in chronic unilateral C6 - C-7 cervical radiculopathy," Annals of King Edward Medical University, vol. 22, no. 2, pp. 102-108, 2016.

[33] A. Ibrahim, N. Fayaz, A. Abdelazeem, and K. Hassan, "The effectiveness of neural mobilization of brachial plexus in patients with chronic unilateral cervical radiculopathy: a single-blinded randomized clinical trial," Bioscience Research, vol. 16, no. 4, pp. 3602-3609, 2019.

[34] I. A. Young, F. Pozzi, J. Dunning, R. Linkonis, and L. A. Michener, "Immediate and short-term effects of thoracic spine manipulation in patients with cervical radiculopathy: a randomized controlled trial," Journal of Orthopaedic \& Sports Physical Therapy, vol. 49, no. 5, pp. 299-309, 2019.

[35] C. Savva, V. Korakakis, M. Efstathiou, and C. Karagiannis, "Cervical traction combined with neural mobilization for patients with cervical radiculopathy: a randomized controlled trial," Journal of Bodywork and Movement Therapies, vol. 26, pp. 279-289, 2021.

[36] P. Langevin, F. Desmeules, M. Lamothe, S. Robitaille, and J.S. Roy, "Comparison of 2 manual therapy and exercise protocols for cervical radiculopathy: a randomized clinical trial evaluating short-term effects," The Journal of Orthopaedic and Sports Physical Therapy, vol. 45, no. 1, pp. 4-17, 2015.

[37] X. J. Cui, M. Yao, X. L. Ye et al., "Shi-style cervical manipulations for cervical radiculopathy: a multicenter randomized- controlled clinical trial," Medicine, vol. 96, no. 31, p. e7276, 2017.

[38] D. G. Kim, S. H. Chung, and H. B. Jung, "The effects of neural mobilization on cervical radiculopathy patients' pain, disability, ROM, and deep flexor endurance," Journal of Back and Musculoskeletal Rehabilitation, vol. 30, no. 5, pp. 951-959, 2017.

[39] F. Hassan, M. Osama, A. Ghafoor, and M. Yaqoob, "Effects of oscillatory mobilization as compared to sustained stretch mobilization in the management of cervical radiculopathy: a randomized controlled trial," Journal of Back and Musculoskeletal Rehabilitation, vol. 33, no. 1, pp. 153-158, 2020.

[40] S. Kumar, "A prospective randomized controlled trial of neural mobilization and MacKenzie manipulation in cervical radiculopathy," Indian Journal of Physiotherapy \& Occupational Therapy, vol. 4, no. 3, pp. 69-75, 2010.

[41] R. Prabhkar, "Cervical spinal mobilization versus TENS in the management of cervical radiculopathy: a comparative, experimental and randomized controlled trial," Indian Journal of Physiotherapy \& Occupational Therapy, vol. 5, no. 1, pp. 9599, 2011.

[42] S. R. I. Bukhari, S. Shakil-ur-Rehamn, S. Ahmad, and A. Naeem, "Comparison between effectiveness of mechanical and manual traction combined with mobilization and exercise therapy in patients with cervical radiculopathy," Pakistan Journal of Medical Sciences, vol. 32, no. 1, pp. 31-34, 1969.

[43] S. Shafique, S. Ahmad, and S. Shakil-Ur-Rehman, "Effect of mulligan spinal mobilization with arm movement along with neurodynamics and manual traction in cervical radiculopathy patients: a randomized controlled trial," The Journal of the Pakistan Medical Association, vol. 69, no. 11, pp. 1601-1604, 2019.

[44] K. Khan, "Effectiveness of manual traction and other physiotherapy treatment in the management of painful cervical radiculopathy," International Journal of Physiotherapy, vol. 3, no. 3, pp. 286-290, 2016.

[45] R. S. Wainner, J. M. Fritz, J. J. Irrgang, M. L. Boninger, A. Delitto, and S. Allison, "Reliability and diagnostic accuracy of the clinical examination and patient self-report measures for cervical radiculopathy," Spine (Phila Pa 1976), vol. 28, no. 1, pp. 52-62, 2003.

[46] B. I. Woods and A. S. Hilibrand, "Cervical radiculopathy: Epidemiology, etiology, diagnosis, and treatment," Journal of Spinal Disorders and Techniques, vol. 28, no. 5, pp. E251E259, 2015.

[47] A. B. Schmid, J. D. P. Bland, M. A. Bhat, and D. L. H. Bennett, "The relationship of nerve fibre pathology to sensory function in entrapment neuropathy," Brain, vol. 137, no. 12, pp. 31863199, 2014.

[48] M. O. Shacklock, Clinical Neurodynamics, Elsevier, 2005.

[49] M. W. Coppieters and D. S. Butler, "Do '_sliders_' slide and ' _tensioners_' tension? An analysis of neurodynamic techniques and considerations regarding their application," Manual Therapy, vol. 13, no. 3, pp. 213-221, 2008.

[50] M. Rade, M. Shacklock, M. Könönen et al., "Normal multiplanar movement of the spinal cord during unilateral and bilateral straight leg raise: quantification, mechanisms, and overview," Journal of Orthopaedic Research, vol. 35, no. 6, pp. 1335-1342, 2017.

[51] E. Thoomes, R. Ellis, A. Dilley, D. Falla, and M. Thoomes-de Graaf, "Excursion of the median nerve during a contra- 
lateral cervical lateral glide movement in people with and without cervical radiculopathy," Musculoskeletal Science \& Practice, vol. 52, p. 102349, 2021.

[52] T. C. Hoffmann, P. P. Glasziou, I. Boutron et al., "Better reporting of interventions: template for intervention description and replication (TIDieR) checklist and guide," $B M J$, vol. 348, no. mar07 3, pp. 1-28, 2014.

[53] F. R. Gärtner, J. Marinus, W. B. van den Hout, C. VleggeertLankamp, and A. M. Stiggelbout, "The cervical radiculopathy impact scale: development and evaluation of a new functional outcome measure for cervical radicular syndrome," Disability and Rehabilitation, vol. 42, no. 13, pp. 1894-1905, 2020. 